\title{
Vision sensor application for intelligent polishing robotic cell
}

\begin{abstract}
Robotics especially on polishing process has not been exploited successfully in manufacturing, despite tremendous research efforts and potential needs in industries. There is still much concern on how robots are used in industries and stumbling blocks in the deployment of technology. In this study, the techniques to alleviate difficulties in using robots and deploying advanced technologies in the real manufacturing environment will be developed. However, the mirror polishing process still depends on human expert, compared to CNC and CAD/CAM technology. On top of that, undesirable working condition exists due to dust and noise. Next, it is quite difficult to find skilled technicians where existing industrial robot (normally with repetitive accuracy of $0.1 \mathrm{~mm}$ ) not suitable for mirror polishing task requirement $(10 \mathrm{~nm})$. This study addresses idea to develop and automate the polishing process by using robots. It applies specifically on flat steel surfaces and the image of the workpiece is captured by vision sensor. The image captured is extracted to develop ANFIS (Adaptive Neuro Fuzzy Inference System) to determine the roughness so that correct polishing parameter will be applied on the surface of the workpiece when polishing is done. ANFIS is well suited to the management of uncertainty, is introduced to address the uncertainty problem associated with surface roughness.
\end{abstract}

Keyword: Vision; Intelligent; Robotic; Polishing 